\title{
V011 STOCHASTIC RESERVOIR CHARACTERIZATION USING AVO INVERSION
}

\author{
A. BULAND', O. HANSEN', P. ABRAHAMSEN ${ }^{2}$ and A. HEKTOEN ${ }^{2}$ \\ IKU Petroleum Research, 7034 Trondheim, Norway \\ ${ }^{2}$ Norwegian Computing Centre
}

\section{Introduction}

Several attempts to utilize information from seismic data for stochastic reservoir characterization have been published, see references in Abrahamsen et al. (1996). To our knowledge none of them consider prestack data. Poststack data contain information on acoustic impedances whereas prestack data contain information on pressure- and shear wave velocities as well as density. This additional information can be used to improve the relationship between seismic data and petrophysical parameters.

A stratigraphic elastic AVO inversion scheme has been developed and the method has been applied to several seismic lines from the North Sea, see Buland et al., 1995, 1996 and Landrøet al. 1995. The inversion method is target-oriented, and P- and S-wave velocities and densities are estimated in a multilayered target zone from the seismic data. The inversion is carried out in the $\tau-p$ domain, which gives an effective forward modelling algorithm making it possible to process $3-\mathrm{D}$ datasets.

A stochastic model, based on correlated Gaussian random fields, has been established linking the seismic data and the petrophysical parameters. Empiric relationships are established by statistical analysis of well data.

A 3-D synthetic rock model of a shallow marine porous reservoir has been developed and a multi-offset seismic survey has been modelled. These data have then been used to test the algorithm.

\section{3-D prestack inversion}

P- and S-wave velocities and densities are estimated in a layered target zone by minimizing a least squares misfit function. A damped Gauss-Newton algorithm is used to find a model for the target zone which minimizes the difference between the modelled and the real seismic data.

It is crucial for 3-D AVO inversion to use an effective forward modelling algorithm. This is obtained in the $\tau-p$ domain by convolving the directional source signature with the reflectivity. $\mathrm{P}$-wave reflections from interfaces in the target zone are modeled, and the corresponding water bottom multiples may be modeled up to a given order.

The results from the $3-\mathrm{D}$ inversion are optimized layer parameters calculated independently in each bin location. The true model and the estimated $\mathrm{P}$-wave velocity, S-wave velocity and density are shown in Figure 1. 


\section{From seismic to reservoir parameters}

The relation between a petrophysical parameter such as porosity or net/gross-ratio, and the inverted seismic parameters are assumed to follow

$$
\text { Petrophysical parameter }=f\left(V_{p}, V_{s}, \rho\right)+\text { error, }
$$

where $f(\cdot)$ must be established from an analysis of well data. The simplest relation is a linear combination of the seismic parameters:

$$
\text { Petrophysical parameter }=\beta_{0}+\beta_{1} \cdot V_{p}+\beta_{2} \cdot V_{s}+\beta_{3} \cdot \rho+\text { error. }
$$

The unknown coefficients can be determined from well data using multiple linear regression. Other relations such as combinations of seismic parameters and use of rock mechanical models have also been investigated.

\section{Stochastic reservoir description}

The challenge is to integrate seismic data and well data, where the seismic data have a much lower vertical resolution than the well data. The inverted seismic parameters and the petrophysical parameter are assumed to be correlated Gaussian random fields. Integration of the different data types can hence be done through kriging and conditional simulation based on the model in (1). Kriging weights the different sources of information and produces 'the most probable' image of the petrophysical parameter given well- and seismic data, see Figure 2. Conditional simulations (Monte Carlo) produces possible realizations of the petrophysical parameters in consistency with the data.

\section{References}

Abrahamsen, P., Hektoen, A., Holden, L. and Munthe, K., 1996, Seismic impedance and porosity: Support effects, Fifth International Geostatistics Congress, Wollongong, Australia.

Buland, A., Landrø M., Andersen, M., and Dahl, T., 1996, AVO inversion of Troll field data, Accepted for publication in Geophysics.

Buland, A., Landrø M., Sollie, R., Andersen, M., and Dahl, T., 1995, Lithology identification by AVO inversion, 65th Ann. Internat. Mtg., Soc. Expl. Geophys.

Landrø, M., Buland, A. and D'Angelo, R., 1995, Target oriented AVO inversion of Valhall and Hod fields data: The Leading Edge, Vol. 14, No. 8, 855-861. 

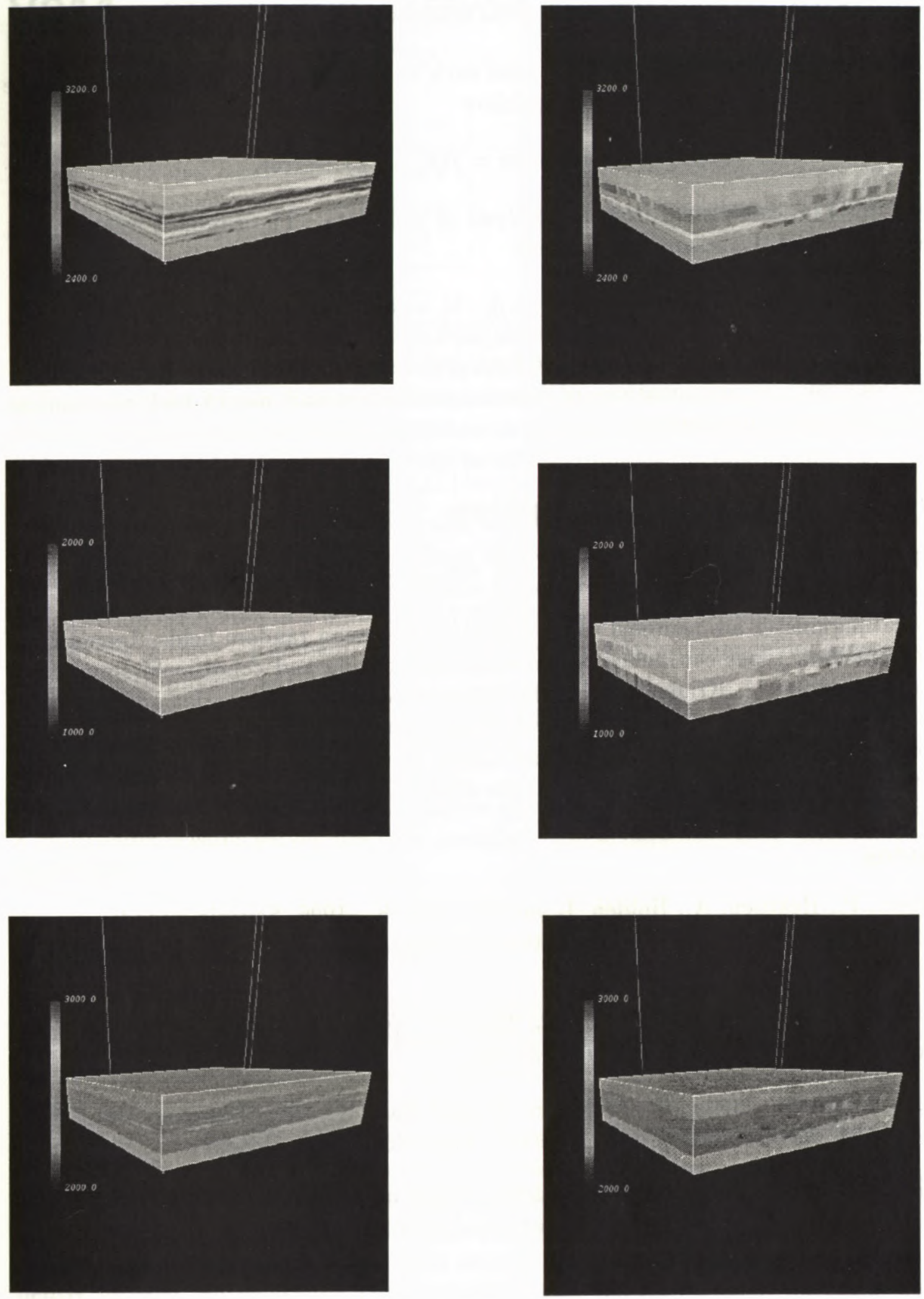

Figure 1: True and inverted P-wave velocity, S-wave velocity and density. 


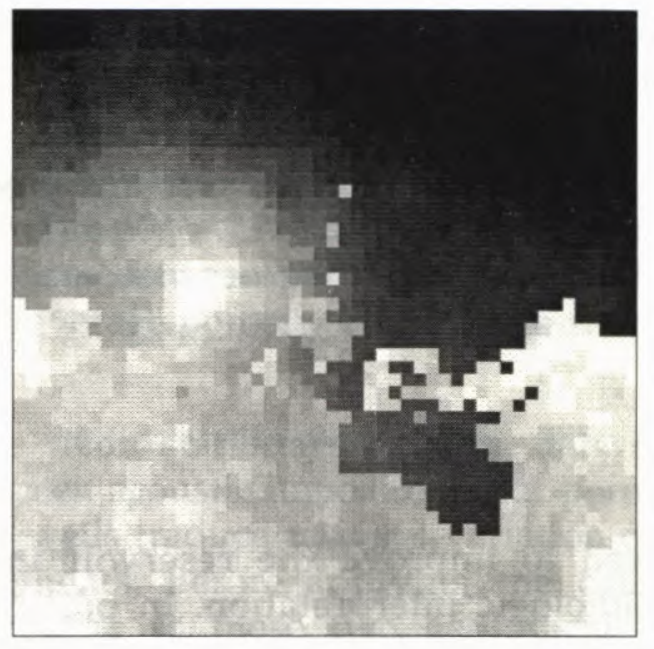

True petrophysical parameter.

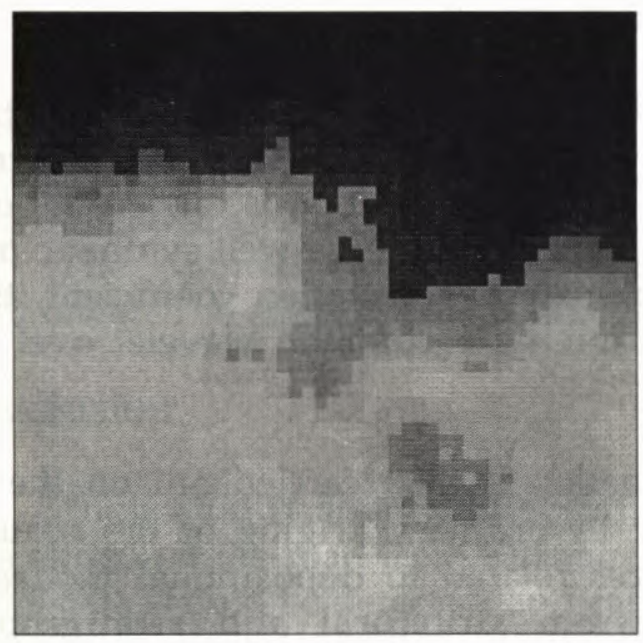

Prediction given seismic parameter.

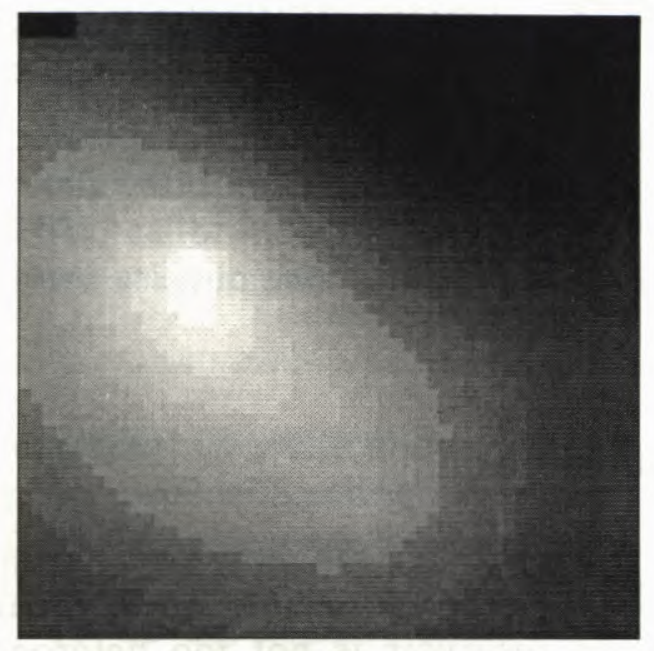

Prediction given well data.

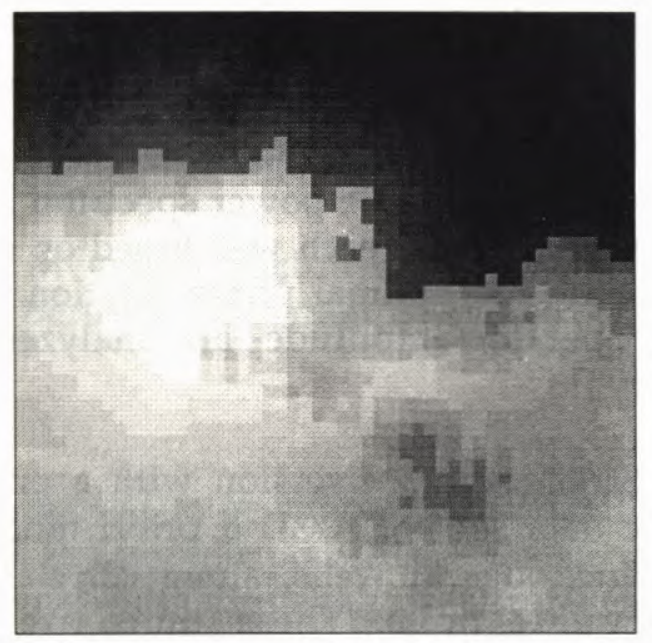

Prediction given data and seismic parameter.

Figure 2: Horizontal cross section through true and predicted petrophysical parameter. 